\title{
High-power single-stage thulium-doped superfluorescent fiber source
}

\author{
Z. Y. Hu P. Yan · Q. Liu $\cdot$ E. C. Ji $\cdot$ Q. R. Xiao $・$ \\ M. L. Gong
}

Received: 6 September 2014 / Accepted: 30 October 2014 / Published online: 7 November 2014

(C) The Author(s) 2014. This article is published with open access at Springerlink.com

\begin{abstract}
In this paper, we report a high-power thulium (Tm)-doped superfluorescent fiber source (SFS) in the $2-\mu \mathrm{m}$ spectral region. The SFS is based on double anglecleaved facet operation and uses a simple single-stage geometry. The copropagating amplified spontaneous emission (ASE) yields a maximum output of $20.7 \mathrm{~W}$ at a center wavelength of $1,960.7 \mathrm{~nm}$, with a full width at half maximum (FWHM) of $\sim 45 \mathrm{~nm}$. The counterpropagating ASE yields a maximum output of $25.2 \mathrm{~W}$ at a center wavelength of $1,948.2 \mathrm{~nm}$, with a FWHM of $\sim 50 \mathrm{~nm}$. The maximum combined output of the SFS is as much as $45.9 \mathrm{~W}$, which corresponds to a slope efficiency of $38.9 \%$. In addition, a model of the $\sim 2 \mu \mathrm{m}$ SFS in Tm-doped silica fibers pumped at $\sim 790 \mathrm{~nm}$ is developed, and the influence of fiber length and end-facet reflectivity on the ASE output performance and the parasitic lasing threshold are studied numerically.
\end{abstract}

\section{Introduction}

Superfluorescent fiber sources (SFSs) have the advantages of broad emission spectra, high output powers, high overall conversion efficiencies, and excellent spatial coherence $[1,2]$. The combination of these characteristics makes SFSs suitable for applications in rotation sensing, pumping of Raman lasers, medical imaging, and optical coherence domain reflectometry $[3,4]$. Therefore, SFSs have become the focus of major research worldwide.

Z. Y. Hu · P. Yan · Q. Liu · E. C. Ji · Q. R. Xiao · M. L. Gong ( $\varangle)$ Center for Photonics and Electronics, Department of Precision Instrument, Tsinghua University, Beijing 100084, China e-mail: gongml@mail.tsinghua.edu.cn

Z. Y. Hu

e-mail: huzhenyue@gmail.com
In the past few years, rapid progress has been made in scaling of the amplified spontaneous emission (ASE) output. For a high-power SFS in the $1-\mu \mathrm{m}$ spectral region [5-9], Schmidt et al. [9] reported a narrow-band SFS with maximum output power of $697 \mathrm{~W}$ at a center wavelength of $1,030 \mathrm{~nm}$ using a two-stage master oscillator power amplifier (MOPA) configuration. In comparison with SFSs in the $1-\mu \mathrm{m}$ spectral region, few studies have been reported to date on high-power broadband thulium (Tm)-doped SFSs in the 2- $\mu \mathrm{m}$ spectral region [10-12]. In 2008, Shen et al. [13] demonstrated a broadband Tm-doped fiber SFS with a single-ended output power of $11 \mathrm{~W}$ using a free-space pump configuration. The slope efficiency was $38 \%$, and the full width at half maximum (FWHM) bandwidth was $36 \mathrm{~nm}$. Recently, Liu et al. [14] reported a 122-W broadband superfluorescence output using a MOPA configuration, with a wavelength range that spanned from 1,935 to 2,075 $\mathrm{nm}$ and a FWHM bandwidth of $25 \mathrm{~nm}$. Obviously, a MOPA scheme can produce a higher ASE output than a single-stage SFS generation scheme. However, the main drawback of the MOPA scheme is that it is rather complex. It requires two kinds of fibers (and their associated pump sources) and one or more Faraday isolators to provide the required degree of attenuation of feedback to both the seed and the amplifier. In contrast, a single-stage SFS generation scheme needs fewer elements and requires less space, so it is both simpler and more compact. In addition, it is not easy to realize an all-fiber SFS for a MOPA scheme because of the destructive effect of the strong backward ASE on the pump source and the other optical components.

In this paper, we report a simple approach for scaling of the output power from a Tm-doped SFS using a singlestage configuration in combination with a double anglecleaved facet geometry to reduce the feedback from the fiber-end facets and provide effective suppression of the 
Fig. 1 Schematic of the Tmdoped SFS setup

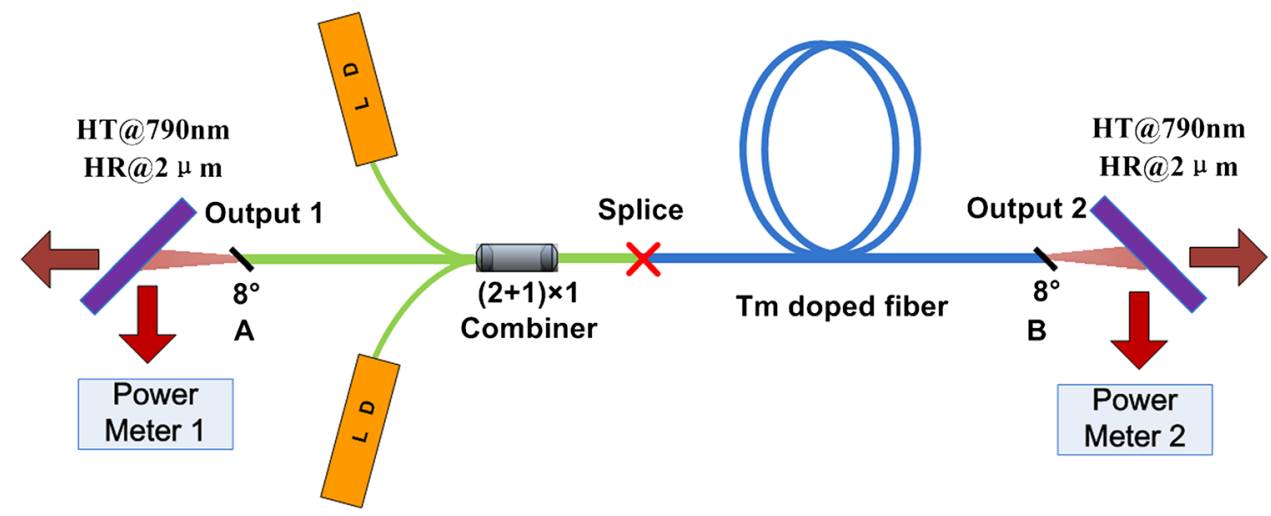

parasitic lasing. With high surface and splicing point quality, the SFS generates maximum output powers of 20.7 and $25.2 \mathrm{~W}$ from the double ends. The maximum combined output of the SFS is as much as $45.9 \mathrm{~W}$, which corresponds to a slope efficiency of $38.9 \%$. To the best of our knowledge, this is by far the highest power produced by a single-stage Tm-doped SFS. Also, a model of the $\sim 2-\mu \mathrm{m}$ SFS using Tm-doped silica fibers pumped at $\sim 790 \mathrm{~nm}$ is developed, and it is interesting to find that both the optical feedback and the active fiber length are of great importance to the power scalability of the SFS. It is shown that multihundred-watt ASEs can be achieved using this scheme.

\section{Experimental setup and results}

The experimental arrangement used for the high-power Tm-doped SFS is shown schematically in Fig. 1. The active fiber of the SFS is a 3-m-long Tm-doped double-clad silica fiber with absorption of $4 \mathrm{~dB} \mathrm{~m}^{-1}$ at $790 \mathrm{~nm}$. The active fiber core has a diameter of $25 \mu \mathrm{m}$ and a numerical aperture (NA) of 0.09 , and the inner cladding has a diameter of $400 \mu \mathrm{m}$ and a NA of 0.46 . Two fiber-pigtailed multimode diodes (LDs) emitting at $790 \mathrm{~nm}$ are used as the pump source with a fiber core diameter of $200 \mu \mathrm{m}$, NA of 0.22 , and total output power of $144 \mathrm{~W}$. In the $(2+1) \times 1$ combiner, which was made in our laboratory, the two input fibers have core and cladding diameters of 200 and $220 \mu \mathrm{m}$, respectively, with an NA of 0.22 . Additionally, the signal fiber has core and cladding diameters of 25 and $400 \mu \mathrm{m}$, respectively. The average coupling efficiency of the two ports is $97.1 \%$. The active fiber and the combiner are cooled to $\sim 10{ }^{\circ} \mathrm{C}$ in a water-cooled heat sink to promote efficient "two-for-one" cross-relaxation.

To obtain the high-power ASE output, the feedback from the fiber-end facets must be reduced. Fresnel reflection is the main physical origin of the optical feedback from the fiber-end facets, and the most common method used to reduce this feedback is the angle-cleaved facet, which

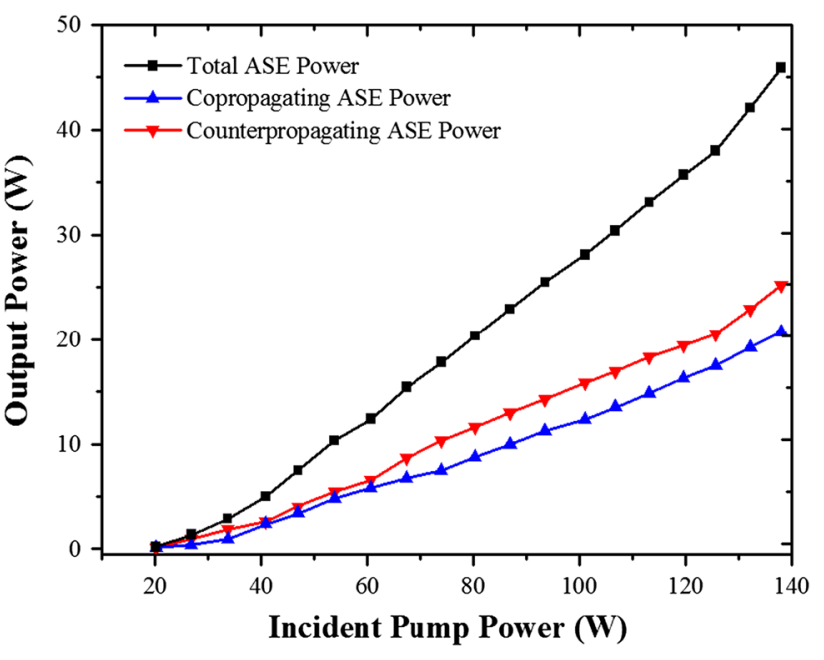

Fig. 2 Experimental ASE output power versus incident pump power for the $\sim 8^{\circ}$ angle-cleaved two-ended configuration

can reduce the end-faced reflectivity to 50-60 dB. In our experiments, the two output ends of the SFS are anglecleaved at $\sim 8^{\circ}$ to produce very low feedback. The fiber end face reflectivity in the absence of defects is approximately $55 \mathrm{~dB}\left(3.16 \times 10^{-6}\right)$ [8]. Scratches and indentations on the core at the fiber-end facets may cause optical performance degradation $[15,16]$. A microscope is therefore used to examine the features of the fiber facet after the anglecleaving process. A fused splice point exists between the combiner and the active fiber, and this splice point would generate serious optical feedback if it was not fused well. In addition, the fiber length is of great importance for the SFS power scalability, and we need to consider both the output power and the efficiency to choose a suitable fiber length; this process is described in the discussion section.

Two dichroic mirrors are used to separate the pump light from the ASE, and the ASE output power is measured using two power meters (Ophir Nova II: FL250A-LP1-SH-V1 and Coherent: Model PM30V1) at each end simultaneously. The ASE output power versus 
Fig. 3 Emission spectra for the Tm-doped SFS: a copropagating ASE, and b counterpropagating ASE

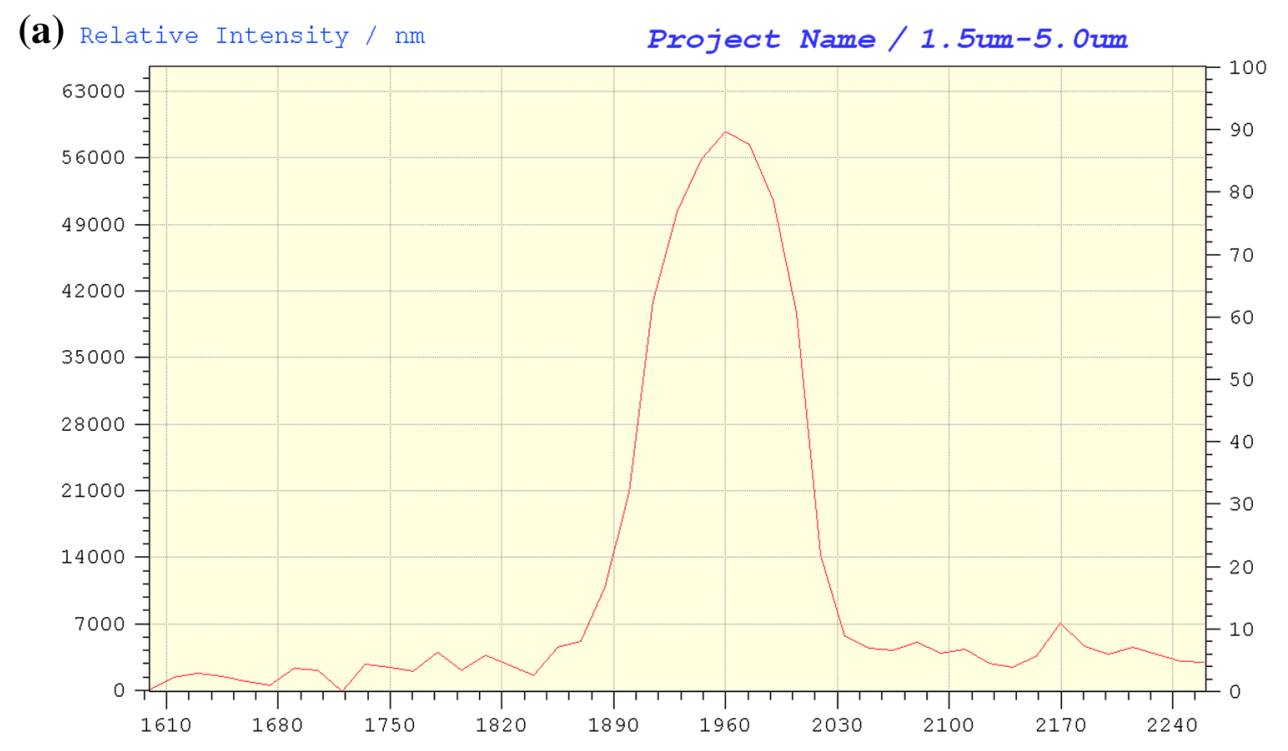

(b)

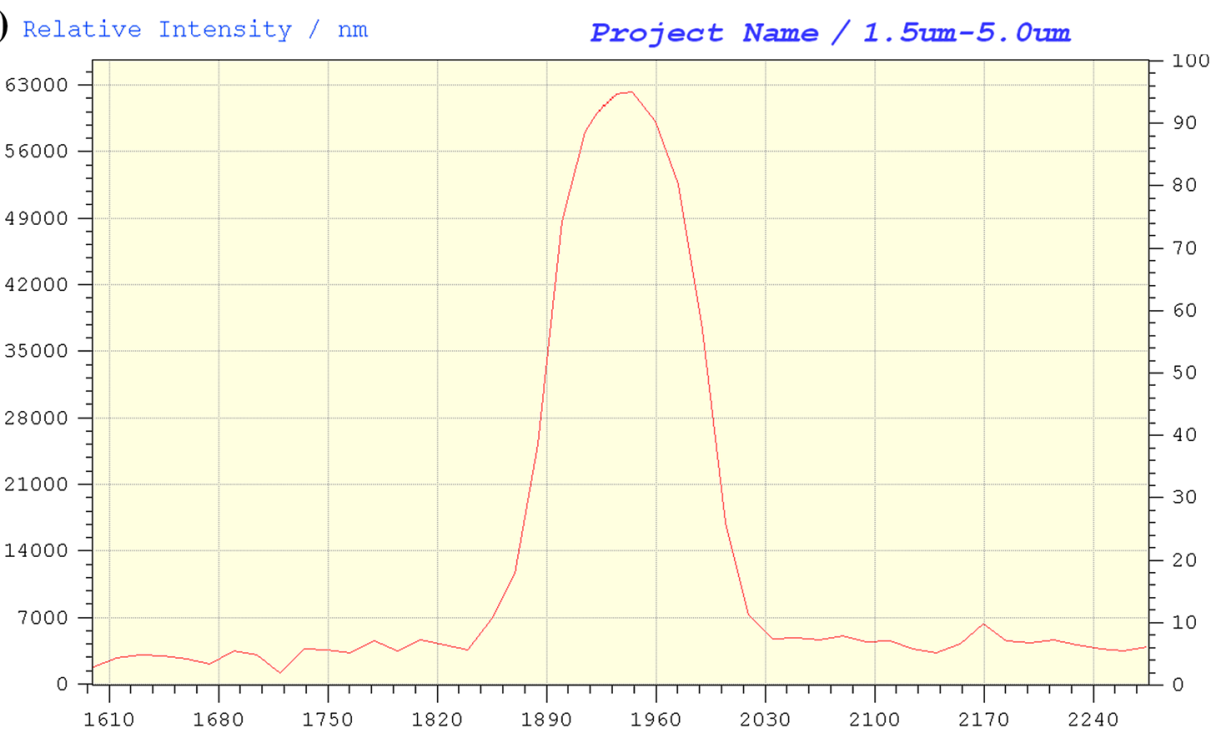

incident pump power characteristics are plotted in Fig. 2. When the pump power is more than $18 \mathrm{~W}$, the SFS begins to generate an ASE output. The maximum ASE output powers for the copropagating (in the same direction as the direction of pump light propagation) and counterpropagating (in the opposite direction to the direction of pump light propagation) beams are 20.7 and $25.2 \mathrm{~W}$, respectively. The total ASE output power is $45.9 \mathrm{~W}$ with an incident pump power of $138 \mathrm{~W}$. The slope efficiencies for the copropagating and counterpropagating beams are 17.5 and $21.4 \%$, respectively. The slope efficiency of the total ASE output power is $38.9 \%$, and the optical-optical efficiency is $33.3 \%$ with an incident pump power of $138 \mathrm{~W}$. In comparison to the copropagating ASE, the counterpropagating ASE has greater slope efficiency. This is because the copropagating ASE is reabsorbed in the low pump-power region, and part of that power is then transformed into the counterpropagating ASE.

The ASE spectra are measured using a spectral analyzer (Spectral Products SM301-EX: CROP35010EV). Note that increasing the pump power will result in the onset of lasing, as confirmed by the sharp peaks in the ASE spectrum. Figure 3 a shows the emission spectrum for the copropagating ASE with an output power of $20.7 \mathrm{~W}$, and Fig. 3b shows the emission spectrum for the counterpropagating ASE with an output power of $25.2 \mathrm{~W}$. The counterpropagating ASE is shown to be blueshifted in comparison to the copropagating ASE. The central wavelength of the copropagating ASE is $1,960.7 \mathrm{~nm}$, spanning from 1,890 to $2,030 \mathrm{~nm}$ with a FWHM of $\sim 45 \mathrm{~nm}$, whereas the central wavelength of the counterpropagating ASE is $1,948.2 \mathrm{~nm}$, spanning from 1,860 to $2,030 \mathrm{~nm}$ with a FWHM of $\sim 50 \mathrm{~nm}$. 


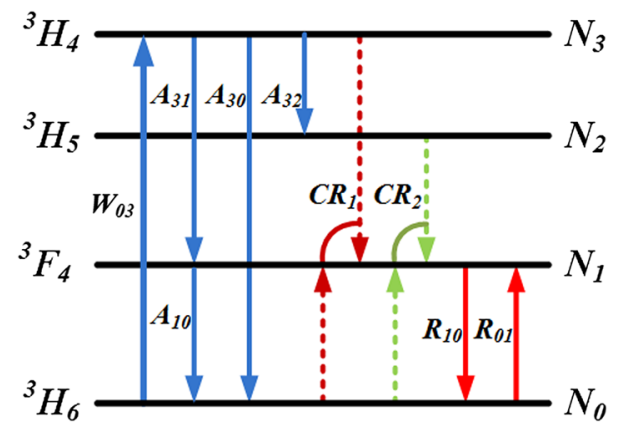

Fig. 4 Schematic view of the four lowest energy levels in the $\mathrm{Tm}^{3+}$ ions

\section{Discussion}

In our experiments, it is shown that a high-power SFS can be realized. Theoretical modeling and simulations are important in investigation of the characteristics of SFSs and subsequent optimization of the SFS parameters for practical systems. A few theoretical models have been reported for the Tm-doped SFS [17-19] without consideration of cross-relaxation (CR). It has been determined that high efficiency can be attained by diode pumping of the ${ }^{3} \mathrm{H}_{6} \rightarrow{ }^{3} \mathrm{H}_{4}$ transition in combination with high Tm-doping concentrations that enable beneficial interionic $\mathrm{CR}$.

In the model, the ${ }^{3} \mathrm{H}_{6} \rightarrow{ }^{3} \mathrm{H}_{4}$ transition is chosen for the pumping scheme, and the energy level diagram of $\mathrm{Tm}^{3+}$ is shown in Fig. 4. Because the lifetime of the ${ }^{3} \mathrm{H}_{5}$ energy level is quite short when compared with the other three energy levels, $N_{2}$ and $\mathrm{CR}_{2}$ both become zero. It is assumed that the pump power is below the laser threshold power, so no laser beams are propagating in the fiber. Based on Jackson's method [20], the rate equations in Tm-doped SFSs can be expressed using the following equations:

$$
\left\{\begin{aligned}
N \approx N_{0} & +N_{1}+N_{3} \\
\frac{\mathrm{d} N_{3}}{\mathrm{~d} t}= & W_{03} N_{0}-\mathrm{CR}_{1} \\
& -\left(A_{32}+A_{31}+A_{30}\right) N_{3} \\
\frac{\mathrm{d} N_{1}}{\mathrm{~d} t}= & A_{31} N_{3}-\left(R_{10}+A_{10}\right) N_{1} \\
& +R_{01} N_{0}+2 \mathrm{CR}_{1} \\
\frac{\mathrm{d} N_{0}}{\mathrm{~d} t}= & \left(R_{10}+A_{10}\right) N_{1}-\mathrm{CR}_{1} \\
& -\left(R_{01}+W_{03}\right) N_{0}+A_{30} N_{3} \\
\mathrm{CR}_{1}= & k_{3101} N_{3} N_{0}-k_{1013} N_{1}^{2}
\end{aligned}\right.
$$

where $N_{0}, N_{1}$, and $N_{3}$ are the population densities of the ${ }^{3} \mathrm{H}_{6},{ }^{3} \mathrm{~F}_{4}$, and ${ }^{3} \mathrm{H}_{4}$ energy levels, respectively ( $N$ is the doping concentration distribution and is assumed to be a constant); $W_{03}$ represents the pumping rate from ${ }^{3} \mathrm{H}_{6}$ to the ${ }^{3} \mathrm{H}_{4}$ energy level; $A_{i j}$ represents the spontaneous transition rate from energy level $i$ to level $j ; R_{i j}$ represents the amplified stimulated transition rate from energy level $i$ to level $j$; and $k_{3101}$ and $k_{1013}$ represent the CR coefficients.

The ASE spectrum is divided into $K$ channels with a central wavelength of $\lambda_{\mathrm{a} i}(i=1,2, \ldots, K)$, and even spacing of $\Delta \lambda$. Therefore, $W_{03}$ and $R_{i j}$ can be defined by:

$$
\left\{\begin{aligned}
W_{03} & =\frac{n \lambda_{\mathrm{p}} \sigma_{\mathrm{a}}\left(\lambda_{\mathrm{p}}\right)}{h c A_{\text {core }}} \Gamma_{\mathrm{p}}\left(P_{\mathrm{p}}^{+}(z)+P_{\mathrm{p}}^{-}(z)\right) \\
R_{10} & =\sum_{i=1}^{K} \frac{n \lambda_{\mathrm{a} i} \sigma_{\mathrm{e}}\left(\lambda_{\mathrm{a} i}\right)}{h c A_{\text {core }}} \Gamma_{\mathrm{a} i}\left(P_{\mathrm{a} i}^{+}(z)+P_{\mathrm{a} i}^{-}(z)\right), \\
R_{01} & =\sum_{i=1}^{K} \frac{n \lambda_{\mathrm{a} i} \sigma_{\mathrm{a}}\left(\lambda_{\mathrm{a} i}\right)}{h c A_{\text {core }}} \Gamma_{\mathrm{a} i}\left(P_{\mathrm{a} i}^{+}(z)+P_{\mathrm{a} i}^{-}(z)\right)
\end{aligned}\right.
$$

where $A_{\text {core }}$ is the cross-sectional area of the core; $n$ is core refractive index; $c$ is the speed of light in a vacuum; $h$ is the Planck constant; $\sigma_{\mathrm{e}, \mathrm{a}}\left(\lambda_{\mathrm{p}}\right)$ are the emission and absorption cross sections of the pump light, respectively; $\sigma_{\mathrm{e}, \mathrm{a}}\left(\lambda_{\mathrm{a} i}\right)$ are the emission and absorption cross sections of the $i$ th ASE light beam, respectively; $\Gamma_{\mathrm{p}, a i}$ are the pump and ASE light power filling distributions, respectively; $P_{\mathrm{p}}^{ \pm}(z)$ are the pump powers in the forward $(z=0$ to $z=L)$ and backward ( $z=L$ to $z=0$ ) directions, respectively; $L$ is the fiber length; and $P_{\mathrm{a} i}^{ \pm}(z)$ are the $i$ th ASE light powers in the forward and backward directions, respectively, and can be described as follows:

$$
\left\{\begin{aligned}
\pm \frac{\mathrm{d} P_{\mathrm{p}}^{ \pm}(z)}{\mathrm{d} z}= & {\left[N_{1} \sigma_{\mathrm{e}}\left(\lambda_{\mathrm{p}}\right)-N_{0} \sigma_{\mathrm{a}}\left(\lambda_{\mathrm{p}}\right)\right] \Gamma_{\mathrm{p}} P_{\mathrm{p}}^{ \pm}(z) } \\
& -\alpha_{\mathrm{p}} P_{\mathrm{p}}^{ \pm}(z) \\
\pm \frac{\mathrm{d} P_{\mathrm{a} i}^{ \pm}(z)}{\mathrm{d} z}= & {\left[N_{1} \sigma_{\mathrm{e}}\left(\lambda_{\mathrm{a} i}\right)-N_{0} \sigma_{\mathrm{a}}\left(\lambda_{\mathrm{a} a}\right)\right] \Gamma_{\mathrm{a} i} P_{\mathrm{a} i}^{ \pm}(z) } \\
& -\alpha_{\mathrm{a} i} P_{\mathrm{a} i}^{ \pm}(z)+2 \sigma_{\mathrm{e}}\left(\lambda_{\mathrm{a} i}\right) N_{1} \frac{h c^{2}}{\lambda_{\mathrm{a} i}^{3}} \Delta \lambda
\end{aligned}\right.
$$

where $\alpha_{\mathrm{p}, a i}$ are the pump and the signal of the $i$ th ASE light loss factors, respectively.

By assuming that the pump light reflectivity at each end of the fiber is zero and that the reflectivity is the same for the whole ASE spectrum, the boundary conditions can be expressed as:

$$
\left\{\begin{array}{l}
P_{\mathrm{p}}^{+}(0)=P_{\mathrm{in}}^{+} \\
P_{\mathrm{p}}^{-}(L)=P_{\mathrm{in}}^{-} \\
P_{\mathrm{a} i}^{+}(0)=R_{1} P_{\mathrm{a} i}^{-}(0) \\
P_{\mathrm{a} i}^{-}(L)=R_{2} P_{\mathrm{a} i}^{+}(L)
\end{array},\right.
$$

in which $P_{\text {in }}^{ \pm}$are the incident pump powers in the forward and backward directions, respectively, and $R_{1}$ and $R_{2}$ 
Fig. 5 Power distributions along the fiber length for the pump, the copropagating ASE, and the counterpropagating ASE for $P_{\text {in }}^{+}=138 \mathrm{~W}$, $R_{1}=R_{2}=55 \mathrm{~dB}$, and $L=3 \mathrm{~m}$

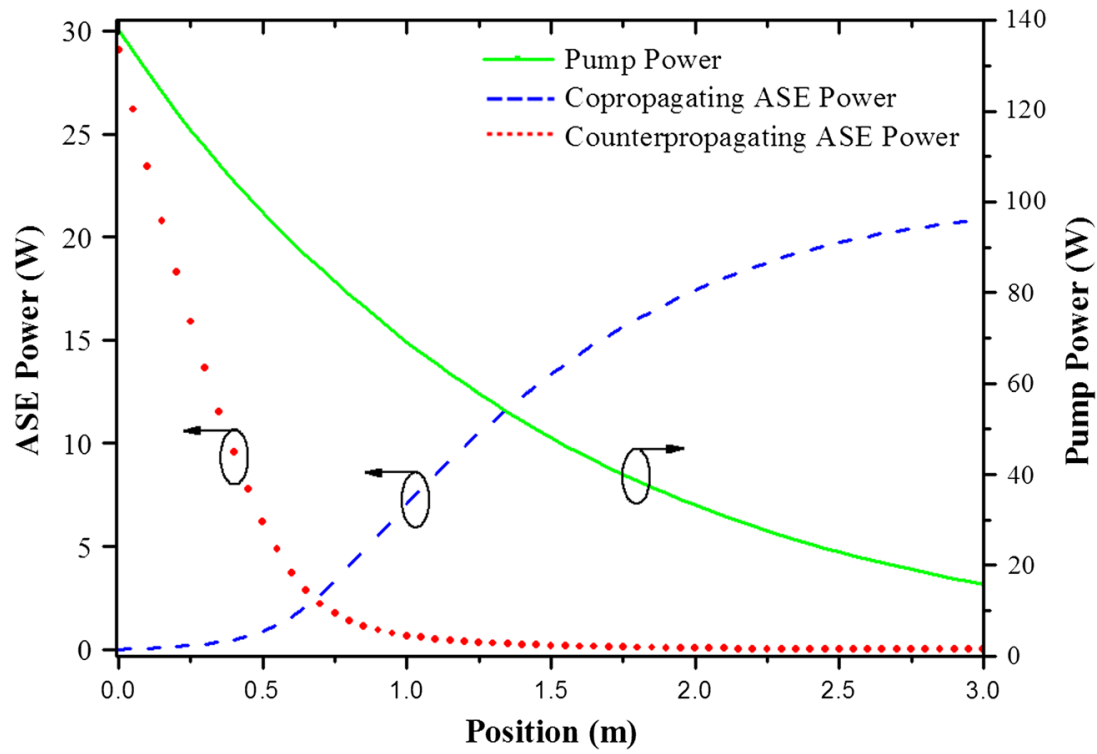

are the reflectivities of the ASE light at $z=0$ and $z=L$, respectively.

For a continuous-wave ASE system, the population densities of each energy level remain steady, and thus, $\frac{\mathrm{d} N_{3}}{\mathrm{~d} t}=\frac{\mathrm{d} N_{1}}{\mathrm{~d} t}=\frac{\mathrm{d} N_{0}}{\mathrm{~d} t}=0$ in (1). Based on the theoretical ASE model composed of (1)-(3), and boundary conditions of (4), we can obtain individual power and population density distributions along the axial direction by discretization into frequency and spatial intervals and performing subsequent iterations until satisfactory convergence of the output parameter occurs by using the fourth-order RungeKutta method. The frequency interval is chosen such that it spans the whole measured cross section of data between the corresponding wavelengths of 1,600 and 2,300 nm [21], which means that the spectral resolution of the calculation is approximately $1 \mathrm{~nm}$. In this paper, we assume that both ends of the fiber are processed by the same method, that the reflectivities of the two ends are equal, and that the pump light only propagates in the forward direction. Results for backward pumping and bidirectional pumping regimes and for different reflectivities at the fiber ends can be obtained in a similar manner. The parameters used in the simulation are listed as follows: $\lambda_{\mathrm{p}}=790 \mathrm{~nm}, c=3 \times 10^{8} \mathrm{~m} \mathrm{~s}^{-1}$, $n=1.458, h=6.626 \times 10^{-34} \mathrm{~J} \mathrm{~s}, A_{\text {core }}=491 \mu \mathrm{m}^{2}$, $\Gamma_{\mathrm{a} i}=0.8, \Gamma_{\mathrm{p}}=3.9 \times 10^{-3}, P_{\mathrm{in}}^{-}=0, \alpha_{\mathrm{p}}=1.2 \times 10^{-2} \mathrm{~m}^{-1}$, $\alpha_{\mathrm{a} i}=2.3 \times 10^{-2} \mathrm{~m}^{-1}, k_{3101}=3 \times 10^{-23}, k_{1013}=0.084 k_{3101}$, $\mathrm{N}=3.54 \mathrm{wt} \%, A_{32}=9.86 \mathrm{~ms}^{-1}, A_{31}=50.7 \mathrm{~ms}^{-1}$, $A_{30}=9.86 \mathrm{~ms}^{-1}$, and $A_{10}=2.99 \mathrm{~ms}^{-1}$. Use of $100 \mathrm{sam}-$ ples along the length of the fiber and $<1,000$ iteration steps can produce accurate results, and the execution only takes a few minutes on a personal computer.

When the incident pump power is $138 \mathrm{~W}$, the reflectivity at each end is $55 \mathrm{~dB}$, and the fiber length is $3 \mathrm{~m}$, the resulting pump and ASE power distributions along the length of the fiber are as shown in Fig. 5. For the counterpropagating case, the power shows a nearly exponential increase, with a maximum power of $29 \mathrm{~W}$ at the output facet $(z=0)$. In the copropagating case, the power emerges with an exponential-like rise at $z<1 \mathrm{~m}$ and then increases slowly to its maximum output of $20 \mathrm{~W}$ at $\mathrm{z}=3 \mathrm{~m}$. The reason for this behavior is that the pump power is not large enough in the appropriate region along the fiber, so both the inverted population and the gain decrease, and as a result, the ASE power increases slowly. The total ASE output is $49 \mathrm{~W}$, which is roughly commensurate with our experimental result of $45.9 \mathrm{~W}$.

As mentioned above, the lasing threshold level determines the maximum available output from an ASE source. To obtain a high-power ASE output, it is therefore necessary to predict the lasing threshold. Based on our SFS model, the frequency-dependent gain can be calculated, and then, the maximum of the frequency-dependent gain is compared with the cavity loss. If these values are equal, this means that the SFS is above the lasing threshold [1, 8]. For the given Tm-doped fiber, the lasing threshold is mainly determined by the reflection at the fiber ends and the fiber length.

First, we assume that the fiber length is finite and that the reflectivity is the same at both ends. To obtain the maximum ASE output with different reflectivities at the fiber ends, we calculate the maximum pump power when lasing is generated. Figure 6 shows the maximum pump power at $L=3 \mathrm{~m}$ with different reflectivities at the fiber ends. Obviously, the maximum pump power increases with decreasing reflectivity. Within the limit, parasitic lasing would not be generated under any circumstances. When the reflectivity is 


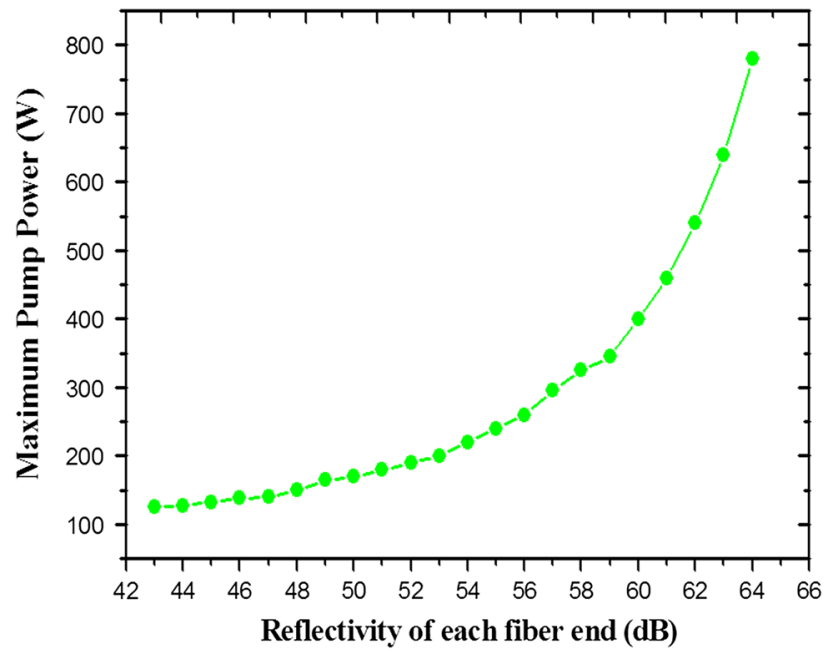

Fig. 6 Maximum pump power versus reflectivity of each fiber end for $L=3 \mathrm{~m}$

$55 \mathrm{~dB}$, the maximum pump power is approximately $235 \mathrm{~W}$, and this power is considerably larger than our experimental maximum pump power of $138 \mathrm{~W}$.

Second, because of the absorption and loss of the signal and pump powers in the fiber, the maximum pump power and the ASE output will change when the fiber length changes. The effect of the fiber length on the ASE source is shown in Fig. 7, assuming that the reflectivity at each fiber end is $55 \mathrm{~dB}$. In general, the maximum pump power decreases when the fiber length increases. However, the maximum pump power increases slowly when the fiber length is longer than $11 \mathrm{~m}$. Because the pump power is not high enough at the far pump end, the ASE self-absorption becomes serious. Also, the ASE output power and the relevant optical-optical efficiency at the maximum pump power are plotted for different fiber lengths. The maximum ASE output could be as high as $142 \mathrm{~W}$ when the fiber length is $1 \mathrm{~m}$, but the optical-optical efficiency is just $23.2 \%$. When the fiber length increases from 5 to $9 \mathrm{~m}$, the efficiency is relatively high and changes slowly at about $42-45 \%$, but the maximum ASE output is just $42 \mathrm{~W}$. The fiber length is therefore chosen to be $3 \mathrm{~m}$ by considering the ASE output and the efficiency in the presented experiment.

\section{Conclusion}

In this paper, we have demonstrated broadband ASE in the $2-\mu \mathrm{m}$ spectral region based on a Tm-doped doubleclad silica fiber, with a single-stage double angle-cleaved facet configuration. The maximum powers for the counterpropagating and copropagating beams are 25.2 and $20.7 \mathrm{~W}$, respectively, with an incident pump power of $138 \mathrm{~W}$. The total ASE output power from the fiber is $45.9 \mathrm{~W}$, which corresponds to a total optical-optical efficiency of $33.3 \%$ and a slope efficiency of $38.9 \%$.

By solving a set of frequency-dependent rate equations, a model of the ASE system is developed, the power distributions along the fiber length for the pump, the copropagating ASE and the counterpropagating ASE are plotted, and the theoretical ASE output is found to be roughly commensurate with our experimental result. For the given fiber, the influences of the fiber facet reflectivity and the fiber length on the laser threshold are discussed, and we can see that these parameters play important roles in the ASE output.
Fig. 7 Maximum pump power, maximum ASE output, and optical-optical efficiency versus fiber length for $R_{1}=R_{2}=55 \mathrm{~dB}$

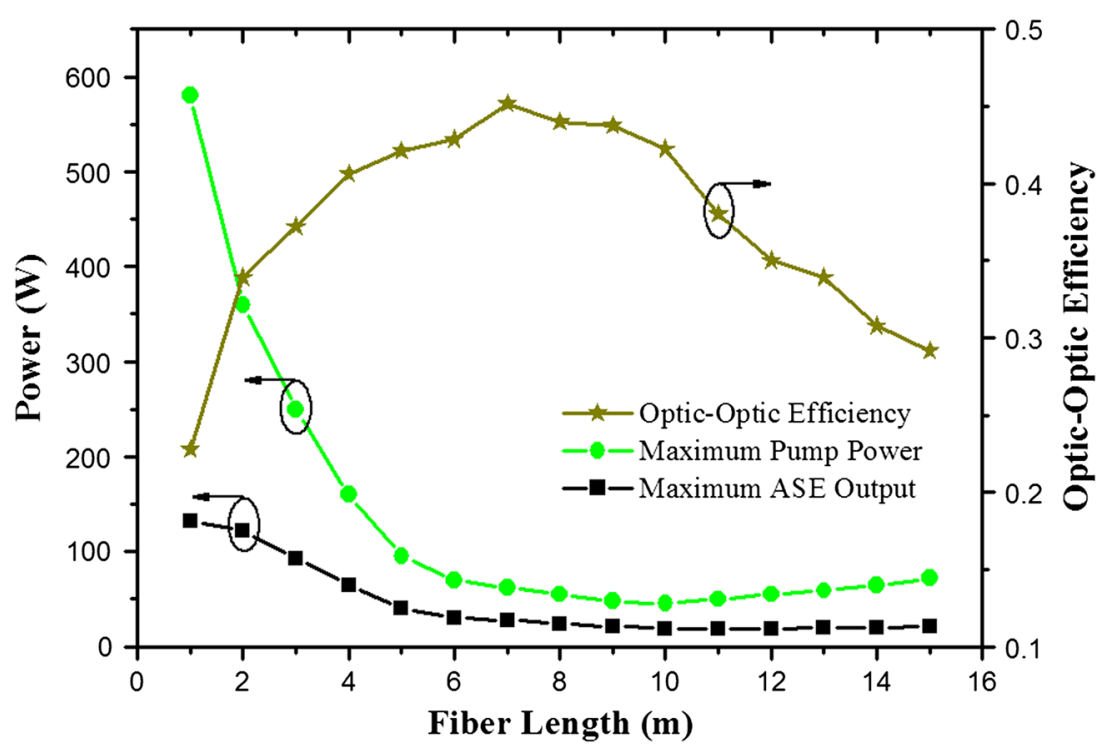


Acknowledgments This research was partially supported by the National Natural Science Foundation of China (Grant No. 61307057), the State Key Laboratory of Tribology, Tsinghua University, China (Grant No. SKLT12B08), and the China Postdoctoral Science Foundation (Grant Nos. 2012M520258 and 2013T60109).

Open Access This article is distributed under the terms of the Creative Commons Attribution License which permits any use, distribution, and reproduction in any medium, provided the original author(s) and the source are credited.

\section{References}

1. I.N. Duling III, R. Moeller, W.K. Burns, C.A. Villarruel, L. Goldberg, E. Snitzer, H. Po, IEEE J. Quantum Electron. 27, 995 (1991)

2. P.F. Moulton, G.A. Rines, E.V. Slobodtchikov, K.F. Wall, G. Frith, B. Samson, A.L.G. Carter, IEEE J. Sel. Top. Quantum Electron. 15, 85 (2009)

3. B.E. Bouma, L.E. Nelson, G.J. Tearney, D.J. Jones, M.E. Brezinski, J.G. Fujimoto, J. Biomed. Opt. 3, 76 (1998)

4. K. Oh, T. Morse, A. Kilian, Meas. Sci. Technol. 9, 1409 (1998)

5. P. Wang, J. Sahu, W. Clarkson, Opt. Lett. 31, 3116 (2006)

6. V. Filippov, Y. Chamorovskii, J. Kerttula, K. Golant, M. Pessa, O. Okhotnikov, Opt. Express 16, 1929 (2008)

7. S.C. Tsai, T.C. Tsai, P.C. Law, Y.K. Chen, IEEE Photonics Technol. Lett. 15, 197 (2003)
8. Q.R. Xiao, P. Yan, Y.P. Wang, J.P. Hao, M.L. Gong, Appl. Opt. 50, 1164 (2011)

9. O. Schmidt, M. Rekas, C. Wirth, J. Rothhardt, S. Rhein, A. Kliner, M. Strecker, T. Schreiber, J. Limpert, R. Eberhardt, Opt. Express 19, 4421 (2011)

10. Y.H. Tsang, T.A. King, D.K. Ko, J. Lee, J. Mod. Opt. 53, 991 (2006)

11. A. Halder, M.C. Paul, N. Shahabuddin, S. Harun, N. Saidin, S. Damanhuri, H. Ahmad, S. Das, M. Pal, S.K. Bhadra, IEEE Photonics J. 4, 14 (2012)

12. Z. Li, A. Heidt, N. Simakov, Y. Jung, J. Daniel, S. Alam, D. Richardson, Opt. Express 21, 26450 (2013)

13. D. Shen, L. Pearson, P. Wang, J. Sahu, W. Clarkson, Opt. Express 16, 11021 (2008)

14. J. Liu, K. Liu, F.Z. Tan, P. Wang, IEEE J. Sel. Top. Quantum Electron. 20, 1 (2014)

15. E. Avram, W. Mahmood, M. Ozer, J. Lightwave Technol. 20, 634 (2002)

16. P. Chanclou, M. Thual, J. Iostec, Appl. Opt. 40, 458 (2001)

17. K. Oh, A. Kilian, P. Weber, L. Reinhart, Q. Zhang, T. Morse, Opt. Lett. 19, 1131 (1994)

18. P. Peterka, B. Faure, W. Blanc, M. Karasek, B. Dussardier, Opt. Quantum Electron. 36, 201 (2004)

19. M. Gorjan, T. North, M. Rochette, J. Opt. Soc. Am. B 29, 2886 (2012)

20. S.D. Jackson, T.A. King, J. Lightwave Technol. 17, 948 (1999)

21. S.D. Jackson, Proc. SPIE 7686, 768608 (2010) 